\title{
First-principles time-dependent simulation of laser assisted desorption of hydrogen atoms from $\mathrm{H}-\mathrm{Si}(111)$ surface
}

\author{
Sergiy Bubin ${ }^{\text {a) }}$ and Kálmán Varga \\ Department of Physics and Astronomy, Vanderbilt University, Nashville, Tennessee 37235, USA
}

(Received 10 February 2011; accepted 26 March 2011; published online 13 April 2011)

\begin{abstract}
The dynamics of hydrogen desorption from H-terminated silicon surface clusters has been simulated in the framework of real space time-dependent density functional theory complemented with molecular dynamics for ions. It has been demonstrated that by choosing an appropriate frequency and intensity of the laser it is possible to remove the hydrogen layer from the surface without destroying the structure of underlying silicon. At the laser field intensities used in the current study (0.5-2.0 V/Å) the desorption process is notably nonlinear. () 2011 American Institute of Physics. [doi:10.1063/1.3580563]
\end{abstract}

Bond scission by laser pulses is an ideal way to induce and control chemical reactions. ${ }^{1-4}$ Selective control of surface reactions ${ }^{4}$ is especially desired as it allows patterning of reaction areas by creating dangling bonds and fabricating functionalized structures by active manipulation of chemical bonds. ${ }^{5}$ Among the many surfaces explored by experiments, the hydrogen terminated $\mathrm{Si}$ surface is the most studied system. ${ }^{6-9}$

Desorption of atoms from surfaces can be induced by electronic excitations directly by coupling between the laser field and the bond electrons or by indirect mechanisms, where the excitation of the substrate transferred to the desorption coordinates. ${ }^{10-12}$ Desorption of atoms can also be initiated by other forms of energy transfer to the system, e.g., by moving a scanning tunneling microscope (STM) tip close to the surface. ${ }^{10,13}$ The hydrogen-terminated $\mathrm{Si}(111)$ surface is one of the simplest and basic examples of a semiconductor interface. Hydrogen atoms chemisorbed onto dangling bonds of the silicon substrate form a protection layer that prevents oxidation and adsorption of impurities. In spite of its apparent simplicity, the hydrogenation of $\mathrm{H}-\mathrm{Si}(111)$ surface is important for both industrial applications and understanding of fundamental processes and mechanisms, including those that occur in more complicated systems.

In this letter, we will carry out first-principles timedependent simulations of laser assisted hydrogen desorption from a $\mathrm{Si}(111)$ surface. The physical mechanisms behind the laser induced desorption are not fully understood and computer simulations are indispensable to gain physical insights of the problem. The real space real time density functional calculation complemented with Ehrenfest molecular dynamics simulation presented in this letter shows that the hydrogen layer can be removed from a silicon surface with a proper choice of laser parameters. This process, which does not destroy the underlying silicon surface, is highly nonequilibrium and nonlinear. The results of the study enhance our understanding of the dynamics of electrons and atoms in and on surfaces.

Hydrogen desorption from silicon has been subject to various theoretical studies ${ }^{14-18}$ including first-principles time-dependent simulations. ${ }^{19}$ The latter work simulated the

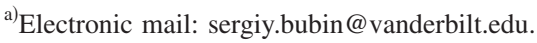

STM induced desorption: The Si-H bond of the hydrogenated $\mathrm{S}(111)$ surface was subjected an excitation by promoting electrons from $\sigma$ to $\sigma^{*}$ orbitals and the motion of atoms was monitored in time after this excitation. In the present work, we add a time-dependent laser pulse to the Hamiltonian of the system and simulate the entire desorption process on the same footing.

The simulations in the present work are carried out in the framework of the time-dependent density functional theory (TDDFT) (Ref. 20) using adiabatic local density approximation with the parameterization of Perdew and Zunger. ${ }^{21}$ Norm-conserving pseudopotentials ${ }^{22}$ were used to represent core electrons. The initial state of the system was prepared by performing the ground state DFT calculation. At the timedevelopment stage we used the following Hamiltonian:

$$
\begin{aligned}
H= & -\frac{\hbar^{2}}{2 m} \nabla_{\mathbf{r}}+V_{\mathrm{a}}(\mathbf{r}, t)+V_{\mathrm{h}}[\rho](\mathbf{r}, t)+V_{\mathrm{xc}}[\rho](\mathbf{r}, t) \\
& +V_{\text {ext }}(\mathbf{r}, t),
\end{aligned}
$$

where $\rho$ is the electronic density, defined as $\rho(\mathbf{r}, t)$ $=\Sigma_{k}\left|\psi_{k}(\mathbf{r}, t)\right|^{2} . V_{\mathrm{a}}, V_{\mathrm{h}}$, and $V_{\mathrm{xc}}$ is the atomic, Hartree, and exchange-correlation potential, respectively. $V_{\text {ext }}$ the explicitly time-dependent potential due to the external electric field. Assuming the direction of the field along the $x$-axis, this potential can be written as $V_{\text {ext }}(\mathbf{r}, t)=x E(t)$. Since the shortest laser wavelength we used $(99 \mathrm{~nm})$ well exceeds the size of the simulations box we assumed $E(t)$ independent on the spatial coordinates at any given instant. The timedependent laser field in our simulations was ramped from 0 to $E_{\max }$ using a Fermi-like envelope $E(t)=E_{\max }(1-[1$ $\left.\left.+e^{(t-a) / b}\right]^{-1}\right) \sin \omega t$, where $a$ and $b$ are parameters controlling the position and smoothness of ramping (we used $a=4.0$ fs and $b=0.75 \mathrm{fs}$ ), and $\omega$ is the frequency of the laser. The maximum amplitude of the laser field, $E_{\max }$, applied in our simulations ranged from 0.5 to $2.0 \mathrm{~V} / \AA$, which corresponds to the intensity of $3.3 \times 10^{16}$ and $5.3 \times 10^{17} \mathrm{~W} / \mathrm{m}^{2}$, respectively. For the time period of $30 \mathrm{fs}$ when the laser is on this corresponds to the range of fluences $50-800 \mathrm{~mJ} / \mathrm{cm}^{2}$ ), which is comparable to the typical values used in experiments. ${ }^{7,9}$

After setting up the initial state, each Kohn-Sham orbital, $\psi_{k}$, was time-propagated using the evolution operator 

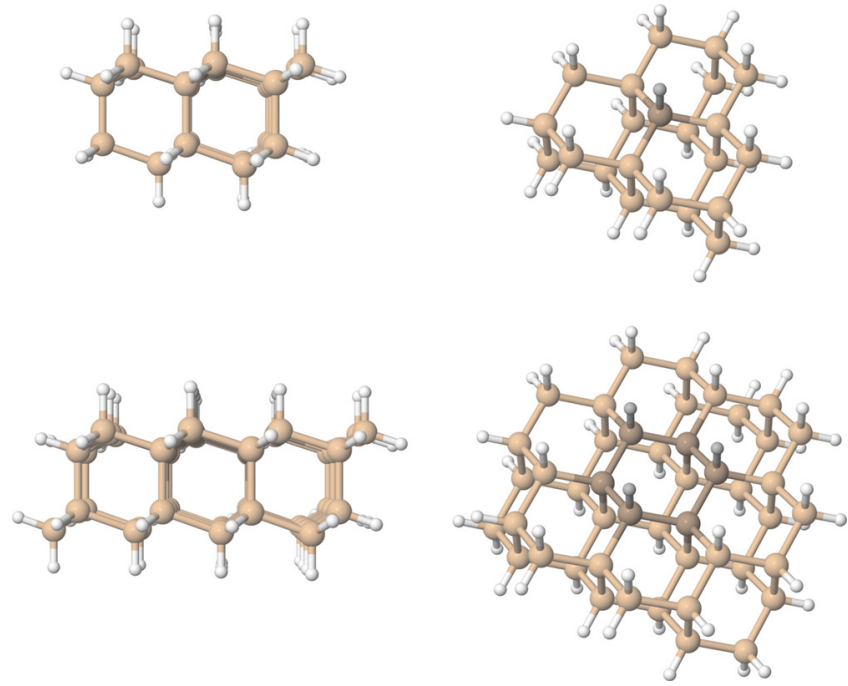

FIG. 1. (Color online) H-Si(111) surface clusters used in present simulations: $\mathrm{Si}_{22} \mathrm{H}_{28}$ (top) and $\mathrm{Si}_{46} \mathrm{H}_{46}$ (bottom). The darkened color indicates the atoms that were allowed to move.

in the form of a fourth order Taylor propagator, ${ }^{23}$

$$
\psi_{k}(\mathbf{r}, t+\Delta t)=\sum_{n=1}^{4} \frac{(i \hbar)^{n}}{n !} H^{n} \psi_{k}(\mathbf{r}, t),
$$

with the time step of $\Delta t=0.001$ fs. The total propagation time was $30 \mathrm{fs}$, which exceeds the typical $\mathrm{Si}-\mathrm{H}$ stretching period (around $8 \mathrm{fs}$ ). All orbitals and the electronic density were represented using a real space grid with spacing of 0.25 $\AA$. To let the electron density oscillate under the laser electric field unobstructively and to leave a sufficient room for the ion dynamics the size of our simulation box was chosen such that the distance from the leftmost/rightmost atom to the simulation box edge in the $x$-direction (in the initial configuration) was not less than $7.7 \AA$. For $y$ - and $z$-direction this distance was $4.8 \AA$ or larger.

The computational cost limits the size of systems in our numerical simulations and we have used finite clusters to model the hydrogenated $\mathrm{Si}(111)$ surface. Two different clusters, $\mathrm{Si}_{22} \mathrm{H}_{28}$ and $\mathrm{Si}_{46} \mathrm{H}_{46}$ (see Fig. 1), have been used in the calculations to evaluate the finite size effects on the results. Both clusters represent four-layer silicon structures. In the present study we used the bond lengths taken from work. ${ }^{24}$ Specifically, the bond lengths between neighboring atoms were as follows ( $\mathrm{Si} j$ stands for the $\mathrm{Si}$ atom in the $j$ th layer): $1.54 \AA$ (H-Si1), $2.331 \AA$ (Si1-Si2), $2.320 \AA$ (Si2-Si3), and $2.352 \AA$ (Si3-Si4). The latter value corresponds to the structure of bulk silicon. Since we used surface clusters of finite size we also had to passivate all dangling $\mathrm{Si}$ bonds on all sides of our structures with hydrogen atoms $(\mathrm{Si}-\mathrm{H}$ bond length of $1.48 \AA$ ).

The dynamics of the ions in our simulations was treated classically within the Ehrenfest scheme. Simulations beyond the classical Ehrenfest trajectories for the ions are an active area of research ${ }^{25}$ but are not very practical at present. In the simulations certain ions were allowed to move under the influence of Hellmann-Feynman forces, while others had fixed positions in order to preserve the general geometric structure of the system under study. In the case of $\mathrm{Si}_{22} \mathrm{H}_{28}$
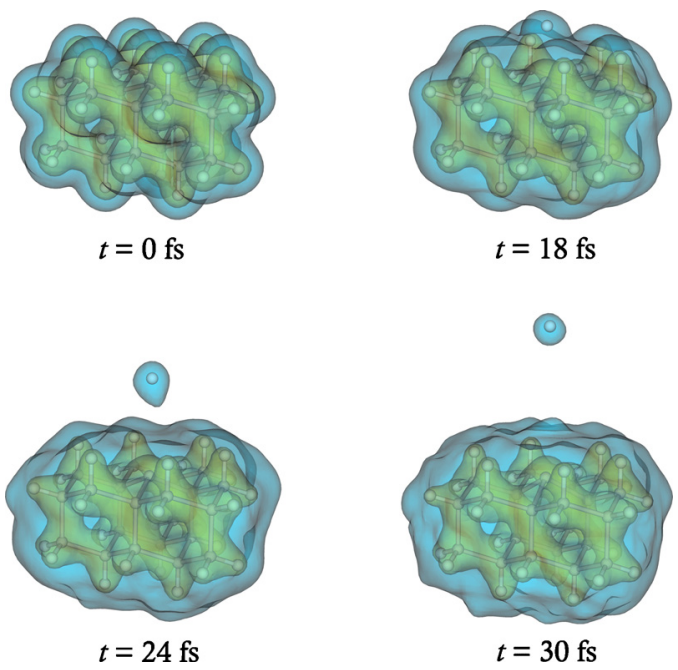

FIG. 2. (Color online) Time-evolution of the electron density in $\mathrm{Si}_{22} \mathrm{H}_{28}$ cluster for the case of $E_{\max }=1.0 \mathrm{~V} / \AA$ and $99 \mathrm{~nm}$ laser wavelength. Two isosurfaces corresponding to the density of 0.15 and $0.015 \AA^{-3}$ are shown. (enhanced online). [URL: http://dx.doi.org/10.1063/1.3580563.1]

cluster only two atoms were allowed to move (a hydrogen atom at the surface and the silicon atom which has a chemical bond with that hydrogen). For the $\mathrm{Si}_{46} \mathrm{H}_{46}$ cluster, out of 92 atoms in the system we let 9 move freely $(3 \mathrm{H}$ atoms, $3 \mathrm{Si}$ atoms in the first atomic plane, and $3 \mathrm{Si}$ atoms in the second atomic plane, as shown in Fig. 1).

When the laser field is turned on the electrons start to oscillate in time in the direction perpendicular to the surface (see Fig. 2). This oscillation induces slow nuclear motion. As the laser field shakes the electron cloud, electron density bubbles burst out from the hydrogen sites and the $\mathrm{Si}-\mathrm{H}$ bond gradually brakes. Figure 2 nicely illustrates the motion of the desorbed hydrogen atom after the bond scission.

A more quantitative picture of hydrogen desorption, the time evolution of the atomic positions, is shown in Fig. 3. Three different laser wave wavelengths $\lambda=99,124$, and 248 $\mathrm{nm}$ (corresponding to $h \nu=5,10$, and $12.5 \mathrm{eV}$ photon energy), are used in the calculations. The $\mathrm{Si}$ clusters, $\mathrm{Si}_{22} \mathrm{H}_{28}$ and $\mathrm{Si}_{46} \mathrm{H}_{46}$, have a $7.5 \mathrm{eV}$ optical gap. For weak laser fields the photon absorption is small for photons whose energy is smaller than the gap and no dissociation should occur. This is corroborated by the results presented in Fig. 3 for $h \nu$ $=5 \mathrm{eV}$ and $E_{\max }=0.5 \mathrm{~V} / \AA$. When the field is weak $\left(E_{\max }\right.$ $=0.5 \mathrm{~V} / \AA$ ) there is no desorption up to $30 \mathrm{fs}$ even for photon energies above the gap. If the laser is turned off, the slightly displaced ions in Fig. 3 would vibrate until their vibrational energy is eventually transferred to the phonon degrees of freedom (for the case of an infinite surface).

Upon increasing the intensity of the laser to $E_{\max }$ $=1.0 \mathrm{~V} / \AA$ the hydrogen gets desorbed in the case of shorter wavelengths (99 and $124 \mathrm{~nm}$ ). As far as the silicon atom is concerned, its $x$-displacements remains small regardless of the wavelength. This suggests that short (20-30 fs) laser pulses of an about $100 \mathrm{~nm}$ laser can be used to strip off hydrogens without changing the structure of the underlying silicon. Experimental observation of desorption of atomic hydrogen from Si surfaces using 157 and $308 \mathrm{~nm}$ lasers has been reported in. ${ }^{7,9}$ It should be noted that in the absence of hydrogens, the clean $\mathrm{Si}(111)$ surface in vacuum eventually evolves into a different reconstruction. 


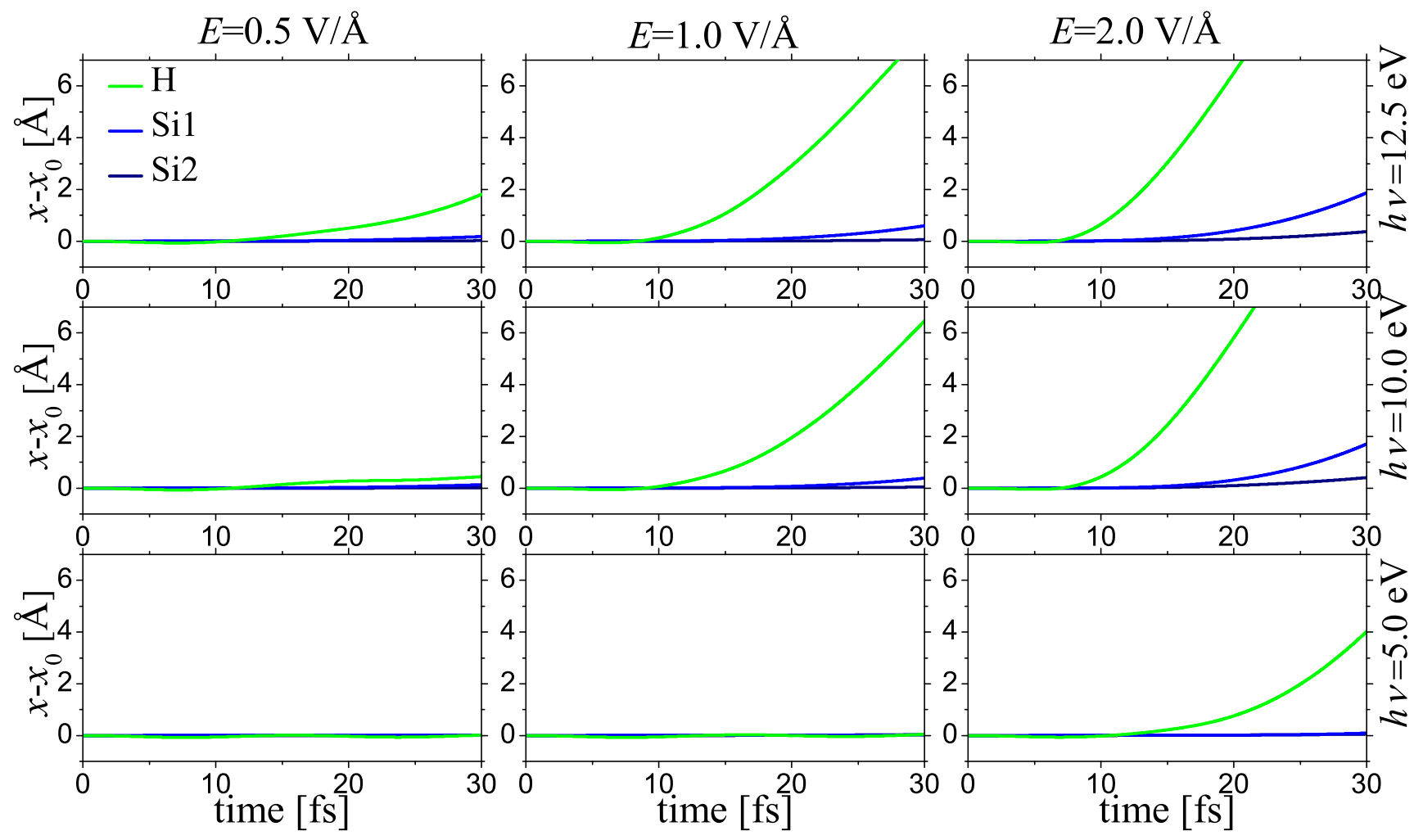

FIG. 3. (Color online) Time-evolution of the $x$-displacement (i.e., perpendicular to the surface) of $\mathrm{H}$ and $\mathrm{Si}$ atoms for various laser field amplitudes and frequencies in the case of $\mathrm{Si}_{46} \mathrm{H}_{46}$ cluster. $\mathrm{Si} 1$ and $\mathrm{Si} 2$ denote atoms in different atomic planes. $\mathrm{Si} 1$ is the closest one to the desorbed $\mathrm{H}$ atom.

At the highest amplitude of the applied laser field $\left(E_{\max }=2.0 \mathrm{~V} / \AA\right)$ the dissociation occurs even for $5.0 \mathrm{eV}$ photon energy. This indicates that we are in the nonlinear regime where there is significant photon absorption below the band gap. At this field strength in the case of 10 and 12.5 $\mathrm{eV}$ photon energy, the silicon atoms on the surface start to acquire significant momentum, which may eventually result in breaking their bonds with other neighboring $\mathrm{Si}$ atoms and escaping. This is in agreement with the experimental results of Ref. 6, where laser induced desorption of $\mathrm{Si}$ atoms from clean $\mathrm{Si}$ surface has been observed. For all values of the laser amplitude and wavelength the $\mathrm{Si1}$ atoms in $\mathrm{Si}_{46} \mathrm{H}_{46}$ follow a trajectory close to that of the $\mathrm{Si}$ atom in $\mathrm{Si}_{22} \mathrm{H}_{28}$. At the same time the $x$-displacements of $\mathrm{Si} 2$ atoms in the $\mathrm{Si}_{46} \mathrm{H}_{46}$ cluster are significantly smaller. While we did not impose any symmetry on the ionic motion, the system maintained the $C_{3 v}$ symmetry to a very high degree. The results obtained for $\mathrm{Si}_{46} \mathrm{H}_{46}$ and for $\mathrm{Si}_{22} \mathrm{H}_{28}$ are in good qualitative agreement showing that the size of our clusters is sufficient to extrapolate the results to realistic $\mathrm{H}-\mathrm{Si}(111)$ surface.

In summary, the dynamics of laser induced bond breaking at a hydrogenated silicon surface has been studied using TDDFT. The calculations show that the laser assisted desorption of hydrogen is a highly nonlinear process, and by choosing the appropriate laser parameters the hydrogen layer can be removed without destroying the Si surface.

This work has been supported by National Science Foundation through Grant No. CMMI-0927345.
${ }^{1}$ A. Assion, T. Baumert, M. Bergt, T. Brixner, B. Kiefer, V. Seyfried, M. Strehle, and G. Gerber, Science 282, 919 (1998).

${ }^{2}$ R. J. Levis, G. M. Menkir, and H. Rabitz, Science 292, 709 (2001).

${ }^{3}$ C. Daniel, J. Full, L. González, C. Lupulescu, J. Manz, A. Merli, S. Vajda, and L. Wöste, Science 299, 536 (2003).

${ }^{4}$ Z. Liu, L. C. Feldman, N. H. Tolk, Z. Zhang, and P. I. Cohen, Science 312, 1024 (2006).

${ }^{5}$ M. Müllenborn, K. Birkelund, F. Grey, and S. Madsen, Appl. Phys. Lett. 69, 3013 (1996).

${ }^{6}$ J. Kanasaki, K. Iwata, and K. Tanimura, Phys. Rev. Lett. 82, 644 (1999).

${ }^{7}$ T. Vondrak and X.-Y. Zhu, Phys. Rev. Lett. 82, 1967 (1999).

${ }^{8}$ K. Bobrov, G. Comtet, G. Dujardin, and L. Hellner, Phys. Rev. Lett. 86, 2633 (2001)

${ }^{9}$ A. Pusel, U. Wetterauer, and P. Hess, Phys. Rev. Lett. 81, 645 (1998).

${ }^{10}$ T.-C. Shen, C. Wang, G. C. Abeln, J. R. Tucker, J. W. Lyding, P. Avouris, and R. E. Walkup, Science 268, 1590 (1995).

${ }^{11}$ P. Avouris, R. E. Walkup, A. R. Rossi, T. C. Shen, G. C. Abeln, J. R. Tucker, and J. W. Lyding, Chem. Phys. Lett. 257, 148 (1996).

${ }^{12}$ P. Guyot-Sionnest, P. Dumas, Y. J. Chabal, and G. S. Higashi, Phys. Rev. Lett. 64, 2156 (1990).

${ }^{13}$ R. S. Becker, G. S. Higashi, Y. J. Chabal, and A. J. Becker, Phys. Rev. Lett. 65, 1917 (1990).

${ }^{14}$ G. Boendgen and P. Saalfrank, J. Phys. Chem. B 102, 8029 (1998).

${ }^{15}$ C. J. Wu, I. V. Ionova, and E. A. Carter, Surf. Sci. 295, 64 (1993).

${ }^{16}$ B. Koehler and S. George, Surf. Sci. 248, 158 (1991).

${ }^{17}$ K. W. Kolasinski, Curr. Opin. Solid State Mater. Sci. 8, 353 (2004).

${ }^{18}$ P. J. Hay, R. C. Boehm, J. D. Kress, and R. L. Martin, Surf. Sci. 436, 175 (1999).

${ }^{19}$ Y. Miyamoto and O. Sugino, Phys. Rev. B 62, 2039 (2000).

${ }^{20}$ E. Runge and E. Gross, Phys. Rev. Lett. 52, 997 (1984).

${ }^{21}$ J. P. Perdew and A. Zunger, Phys. Rev. B 23, 5048 (1981).

${ }^{22}$ N. Troullier and J. L. Martins, Phys. Rev. B 43, 1993 (1991).

${ }^{23}$ K. Yabana and G. F. Bertsch, Phys. Rev. B 54, 4484 (1996).

${ }^{24}$ E. Kaxiras and J. D. Joannopoulos, Phys. Rev. B 37, 8842 (1988).

${ }^{25}$ A. Abedi, N. T. Maitra, and E. K. U. Gross, Phys. Rev. Lett. 105, 123002 (2010). 